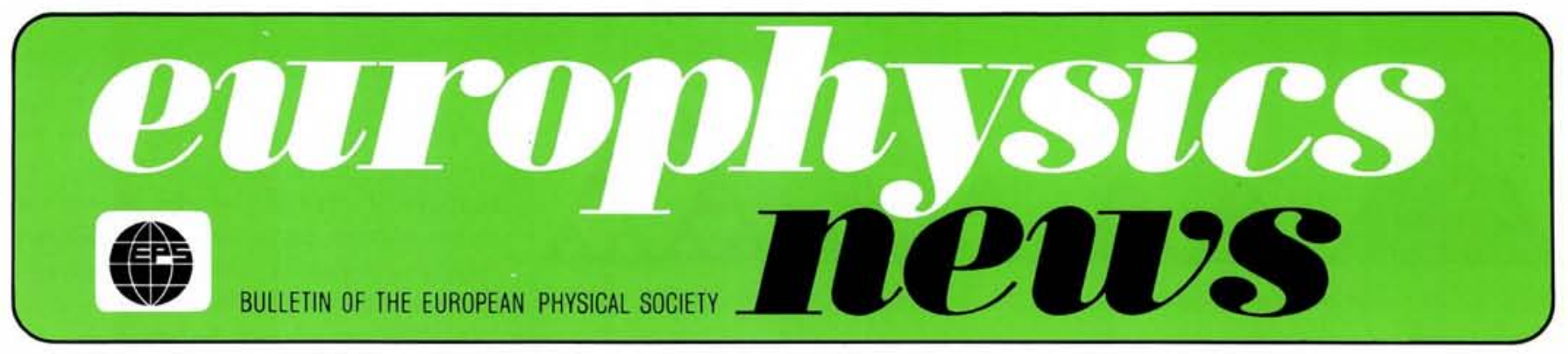

J.A.

\title{
Fractals in Physics
}

\section{Amnon Aharony, Tel Aviv}

(School of Physics and Astronomy, Tel Aviv University)

Many structures in physics are self-similar, i.e. invariant under scale transformation. Mandelbrot's fractal geometry is a powerful tool to study the influence of the geometry on the physics, and to construct fractal models for physical systems.

Benoit Mandelbrot was the first to realize that many shapes in Nature, ranging from trees, coastlines, rivers, clouds, lungs, etc. to graphs describing economic phenomena, exhibit self-similar features ${ }^{1}$ ). This approach has developed into a wide scientific discipline, whose role in physics is reviewed here. The relevant concepts are first introduced via a discussion of percolation clusters, their geometry and their physical properties $\left.{ }^{2}\right)$. Other fractal structures and models are then briefly described. Instead of attempting a detailed list of references, the interested reader is referred to four recent conference proceedings $\left.{ }^{3,4,5,6}\right)$ and to a more technical review ${ }^{7}$ ).

\section{Percolation}

Fig. 1 shows parts of a triangular lattice, in which only a fraction $p=1 / 2$ of the sites are occupied. The occupation of each site is completely random, independent of other sites. This is a specific example of a percolation model. Such models are widely used to describe alloys of materials with different properties, e.g. metal-insulator, metal-superconductor, magnet-non-magnet, etc. For convenience, we shall use the metalinsulator example, and the occupied sites in Fig. 1 refer to metallic atoms. Electric current may flow only between nearest neighbour sites. At low concentrations, small $p$, connected neighbouring sites form only small finite clusters, and a very large sample will have zero conductivity. At $p$ close to unity there always appears a large cluster which connects between the edges of the sample. The size of this cluster diverges to infinity with the sample's size. The conductivity of the sample is thus finite. In the thermodynamic limit, when the sample is infinitely large, there exists a sharp threshold, $p_{c}$, below which the conductivity is zero. Above $p_{c}$ the probability per site to belong to the "infinite" cluster grows as $P_{\infty}(p) \sim\left(p-p_{c}\right)^{\beta}$ and the conductivity $\sigma$ grows as

$$
\sigma(p) \sim\left(p-p_{c}\right)^{\mu}
$$

Both below and above $p_{c}$, the linear size of the finite clusters is rarely larger than the percolation connectedness length $\xi$, which diverges when $p \rightarrow p_{c}$ as $\xi \sim\left|p-p_{c}\right|^{-v}$. The exponents $\beta, \mu, v$, etc. are universal, and depend only on the dimensionality $d$ of the system (and not on the lattice structure, etc.).

At $p=p_{c^{\prime}}$ the probability to belong to the "infinite" cluster $P_{\infty}$ vanishes. However, for a finite sample, of linear size $L$, there is a finite probability to have a large cluster which connects the edges. For the triangular lattice, the percolation threshold turns out to be exactly $p_{c}=$ $1 / 2$. Fig. 1a thus shows a finite percolating sample, at $p_{c^{\prime}}$ and the sites of the largest cluster are connected by bonds.

Consider now a coarse graining of Fig. 1a: We group the sites on the lattice into cells of three sites, and replace each cell by a single "renormalized" site. The new site is occupied if a majority of its components (i.e. two or three) were occupied, and empty otherwise. The resulting new lattice, and its largest cluster,

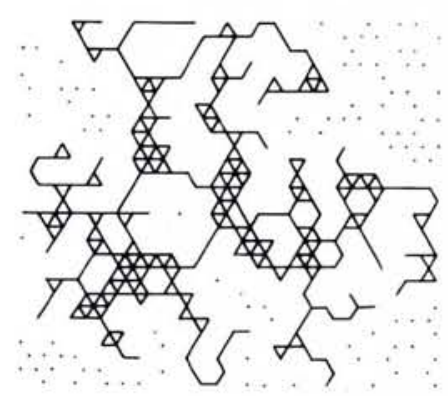

(o)

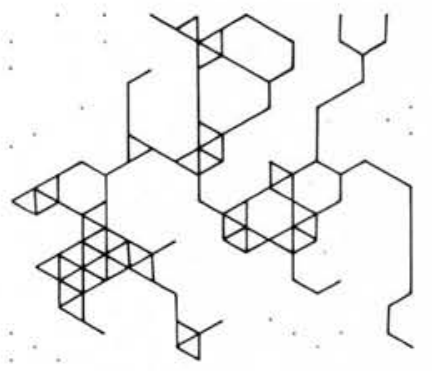

(b)

Fig. 1 - A dilute triangular lattice, at $p=1 / 2$. The sites of the largest cluster are connected by bonds. (a) Original size. (b) Rescaled by $b=\sqrt{ } 3$. Each size replaces a cell of three sites in (a).

\section{Contents}

Fractals in Physics

Artificial Semiconductor Superlattices and Quantum Well Structures

New Members of EPS

The $\mathrm{H}$-Mode of ASDEX

ICTP Donation Programme

Hydrogen Plasma Phase Diagram and Properties

Changes to Constitution

Executive Committee 1986/87

New Collaborating Society

IOM Delegates 

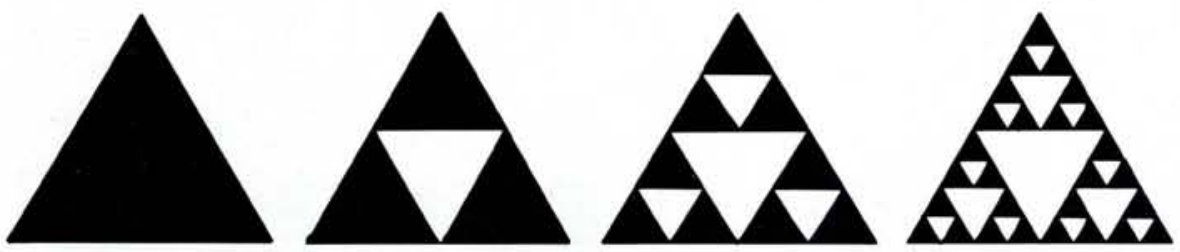

Fig. 2 - Four stages of the Sierpinskii gasket.

are shown in Fig. 1b. Note that Fig. 1b looks exactly like a piece of Fig. 1a, and it is impossible to tell from the picture at what level of "coarse graining", or magnification, the two pictures were taken. This is a qualitative manifestation of self-similarity.

A quantitative measure of this selfsimilarity is obtained as follows: The length of a unit distance between neighbours in Fig. $1 \mathrm{~b}$ is larger than that of Fig. $1 a$ by $b=\sqrt{ } 3$. If we count the number of sites on the largest clusters in the two figures, we find that their ratio is about $2.8 \cong b^{D}$, with the exponent $D \cong 1.9$. This implies that the number of sites on the largest cluster scales with length as

$$
M \sim L^{D}
$$

This should be contrasted with the scaling of a homogeneous system, with a uniform density of sites, which would be $M \sim L^{d}$, where $d$ is the Euclidean dimension of the lattice $(d=2$ in Fig. 1). The non-integer exponent $D$ is called the fractal dimensionality, and self-similar geometrical structures which obey a power law like (1) are the simplest example of fractals. Although alternative definitions of $D$ are available, Eq. (1) is sufficient for the self-similar case.

The coarse graining (or renormalization group) procedure outlined above is equivalent to a measurement of $M(L)$ using different basic measuring yardsticks, as originally used by Mandelbrot to show that the coast of Britain has $D=$ 1.3. An alternative way amounts to measurements of $M(L)$ within different volumes $L^{d}$, around an origin (which belongs to the "infinite" cluster) and plots of $\ln \rho v s \ln L$, for the density $\rho=$ $M / L^{d} \sim L^{D-d}$. If such measurements are repeated for $p>p$ then these plots will have a slope $(D-d)$ for $a<L<\xi$ (a is some microscopic scale), and will approach the constant average uniform density $P_{\infty}$ for $L>\xi$. Matching the two at $L \sim \xi$ identifies the relation:

$$
D=\alpha-\beta / v
$$

as found in the late 1970's by Stanley, Mandelbrot, Kirkpatrick and others.

The difference $(D-d)$ also determines the decay of the correlation function: If the origin belongs to the "infinite" cluster then the probability that a site at a distance $r$ from the origin also belongs to that cluster decays as $r^{\mathrm{D}-\mathrm{d}}$. These power laws have been confirmed both on computer experiments and on real thin metal films. The correlation function is also measured via X-ray or neutron scattering.

At $p=p_{c}, \xi=\infty$ and the only functional form allowed for $M(L)$ is a power law. The same applies to all physical properties: on a self-similar fractal structure they all behave as powers of $L$. The aim of the current research is to identify the relevant exponents, and to attempt to relate them to each other or to calculate them from first principles. In particular, much emphasis has been placed on identifying the geometrical properties which are responsible for the physical behaviour.

\section{Physical Properties}

Consider now the conductivity of the sample in Fig. 1a. Clearly, it will depend only on the "infinite" cluster. However, the classical DC conductivity will not be affected by dangling bonds, which are connected to the edges of the sample via one route only. The remaining part of the cluster, called the "backbone", has a

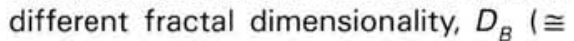
1.7 at $d=2$ ).

Similarly, other physical properties require knowledge of the fractal properties of other subgroups of sites on the cluster. For example, the low temperature decay of spin correlations on a percolating magnetic cluster is determined by the singly connected bonds (the cutting of each of which disconnects the two relevant spins from each other). The number of these scales as $M_{\text {sc }} \sim L^{i s c}$, with $\bar{\zeta}_{\mathrm{sc}}=1 / v(=3 / 4$ at $d=2)$. In contrast, the diamagnetic susceptibility of a cluster will be determined by the total area of loops on it. Another geometrical property involves the external perimeter of the cluster, which governs the motion of electrons at high magnetic fields or the adsorption of new atoms on to the cluster.

Fig. 3 - The Mandelbrot-Given model fractal.
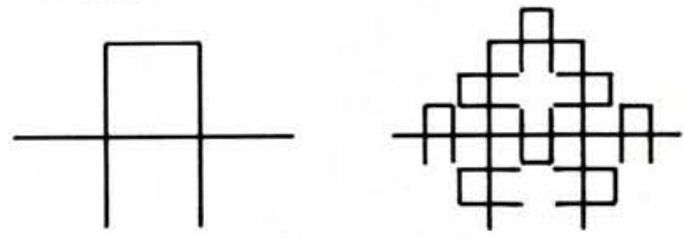

Other properties (e.g. the propagation of fires in dilute forests) are determined by the minimum graph distance, or the "chemical" distance on the cluster. The number of sites (or bonds) on each of these subgroups scales as a different power of the size, $L$, and thus involves a different fractal dimensionality.

The study of the conductivity demands yet another geometrical structure. With uniform systems, the Einstein relation relates the DC conductivity $\sigma$ to the diffusion coefficient $\delta$. A random walker will travel at time $t$ a mean square distance $\left\langle r^{2}\right\rangle \sim \delta t$. The number of steps thus scales as $t \sim r^{d}$, with the fractal dimensionality of the random walk $d_{w}=2$. When the random walk is performed on a fractal structure, like the infinite percolation cluster, $d_{w}$ is no longer equal to two. Instead, $d_{w}=2+\theta$, where $\theta$ describes the decay of $\delta$ with distance, $\delta \sim r^{\theta}$ (this decay is related to that of the conductivity with $L$, similar to that of $P_{\infty}$ described above). Knowledge of $d_{w}$ (or $\theta$ ) determines the conductivity exponent $\mu$, via the Gefen-AharonyAlexander relation

$$
\theta=(\mu-\beta) / v .
$$

When a random walker moves on a fractal structure, the number of sites visited after time $t$ will scale as $S(t) t^{\bar{d} / 2}$, with the "fracton" (or "spectral") dimensionality $\bar{d}=2 D /(2+\theta)$. The geometrical shape of this fractal set of visited sites has therefore been of much recent study. The reference to $\bar{d}$ as a dimensionality comes from the observation of Alexander and Orbach, that $\bar{d}$ replaced $d$ in the frequency dependence of the vibrational density of states, $N(\omega)$ $\omega^{\bar{d}-1}$. Simple arguments about $S(t)$ by Alexander and Orbach, Rammal and Toulouse, Layvraz and Stanley and others, yield $\bar{d}=4 / 3$. This "AlexanderOrbach" value seems to give an excellent (although not exact) estimate for percolation clusters at $d>2$. Away from $p_{c}, N(\omega)$ crosses over rather sharply from the phonon behaviour $\omega^{d-1}$ (at low $\omega)$ to the fracton one, $\omega^{\bar{d}-1}$. Such crossovers are probably observed in some glassy systems.

\section{Fractal Models}

The percolation clusters (Fig. 1), and practically all the fractals which arise in physical applications, are random. The 


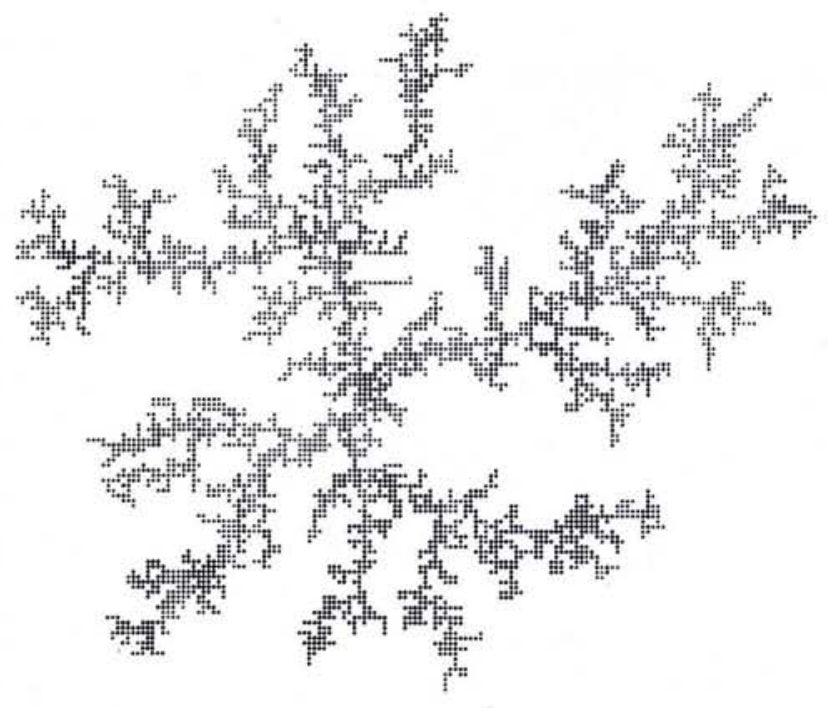

Fig. 4-A diffusion limited aggregation cluster (on a square lattice).

power law (1) is obeyed only on the average. In fact, the mean squared fluctuations about this average, related to Mandelbrot's notion of "lacunarity", scale for percolation clusters as $\triangle M^{2} \sim$ $L^{2 D}$. Similar fluctuations in the resistance are important in determining the noise in these circuits, and are the subject of much recent research. The structure of the various random distributions, and their cumulants, also turns out to involve interesting fractal features.

One disadvantage of this randomness is that it prevents any analytic explicit calculation of physical properties of these structures. In order to understand the influence of the geometry on the physics it has been very useful to study non-random fractal models, which imitate many of the geometrical properties of the physical system. Two such examples are shown in Figs. 2 and 3. Fig. 2 shows four stages of the recursive construction of the Sierpinski gasket: at each iteration, the length scale is divided by $b=2$, and each triangle is replaced by three smaller ones (the central small triangle is cut out). Thus, $3=2^{D}$ and $D$ $=\ln 3 / \ln 2 \cong 1.58$. This structure, and its $d$-dimensional generalizations, were proposed by Gefen, Aharony, Mandelbrot and Kirkpatrick as a model for the backbone of the infinite cluster, and

Fig. 5 - Two stages of the triadic Koch curve. $D=\ln 4 / \ln 3 \cong 1.26$.
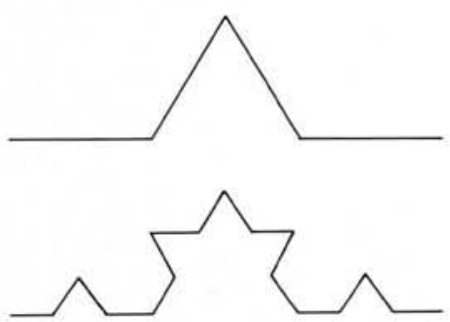

have been widely used to calculate various physical properties, ranging from mass distribution and conductivity to fluctuations and noise, quantum eigenstates of the Schrödinger equation and superconductivity.

Fig. 3 shows a "branching" Koch curve, proposed by Mandelbrot and Given to model the full two dimensional infinite cluster. Again, many physical properties have been easily calculated, and they turn out to be rather close to their numerical simulation and experimental counterparts. For example, $D=\ln 8 / \ln 3 \cong 1.89, D_{\mathrm{B}}=\ln 6 / \ln 3 \cong$ $1.63, \bar{\zeta}_{s c}=\ln 2 / \ln 3 \cong 0.63, \mu / v=$ $\ln (11 / 4) / \ln 3 \cong 0.92$.

\section{More Fractals}

Many additional natural structures exhibit fractal geometries, similar to those described above. For each of these, the physical properties are again determined by the geometry of the appropriate substructures.

Much recent interest has been focussed on structures which arise in aggregation processes. Fig. 4 shows a cluster obtained by diffusion limited aggregation (DLA), first suggested by Witten and Sander: monomers diffuse and stick to the cluster when they reach it. One finds $D \cong 1.7$ in two dimensions and $D \cong 2.4$ in three dimensions. Such models seem to imitate very well the geometry of gold colloids, as measured by electron microscopy and by light scattering by Weitz and collaborators. Similar structures were observed by $X$ ray and by neutron scattering from silica colloid aggregates (by Schaeffer et al. and by Sinha et al.).

Much of the interest in the structure of DLA followed the realization, by Patterson, that viscous fingering, which arises when a high viscosity fluid is displaced by a low viscosity one in a porous medium, is governed by the same continuum Laplace equation. Similar structures are also exhibited by the discharge patterns in dielectric breakdown, as recently discussed by Pietronero and collaborators.

Unlike the percolation clusters (Fig. 1), those of DLA have practically no loops. They grow mainly at their tips, and this determines their structure, as well as their physical properties. Both are currently under intensive study, both theoretically and experimentally.

Other examples of fractals in statistical physics include: polymers, which are described by self avoiding random walks and imitated by non-branching Koch curves (Fig. 5); branching polymers, which are described by "lattice animals", i.e. large percolation clusters in the limit of zero concentration $(p \rightarrow 0)$; noise or error bursts in data transmission, imitated by the Cantor set (Fig. 6), etc.

Fig 6. - Three stages of a Cantor set. $D=$ $\ln 2 / \ln 3 \cong 0.63$.

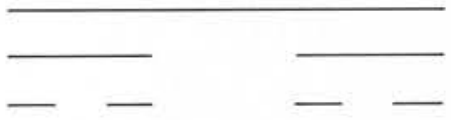

Fractals also occur in hydrodynamics, and a widely studied example involves the fractal geometry of clouds, which is probably directly related to the self-similar nature of fully developed turbulence. Fractal structures arise in geophysics, in maps of cracks following earthquakes, they arise in surface physics, in the geometry of rough surfaces, and in many other (sometimes unexpected) places. Mandelbrot's idea, that Nature's geometry is often fractal, has indeed developed into a very useful scientific discipline.

\section{SUGGESTED READING}

1. Mandelbrot B.B., The Fractal Geometry of Nature (W.H. Freeman, New York) 1982.

2. Stauffer D., Introduction to Percolation Theory (Taylor and Francis, London) 1985. 3. 'Proceedings of a Symposium on Fractals', eds. M.F. Shlesinger, B.B. Mandelbrot and R.J. Rubin, J. Stat. Phys. 36 (1984) 519 921.

4. Kinetics of Aggregation and Gelation, eds. F. Family and D.P. Landau (North- Holland, Amsterdam) 1984.

5. On Growth and Form: Fractal and NonFractal Aspects, eds. H.E. Stanley and N. Ostrowsky (M. Nijhoff-Kleuver) 1985.

6. Fractals in Physics, Proceedings of the 1985 Trieste Conference, eds. L. Pietronero and E. Tosatti (North-Holland, Amsterdam) 1986.

7. Aharony A., 'Fractals in Statistical Physics', Ann. N.Y. Acad. Sci. (1985). 